\title{
Authors reply: Importance of standardisation of HAI definitions in interpretation of international and/or multinational prevalence studies
}

\author{
M J Veldman-Ariesen (Marie-Jose.Veldman@rivm.nl) ${ }^{1}$, Eilers $^{1}$ \\ 1. Mater Misericordiae University Hospital, Dublin, Ireland \\ 2. Health Protection Surveillance Centre, Dublin, Ireland \\ 3. Beaumont Hospital, Dublin, Ireland
}

Citation style for this article:

Veldman-Ariesen MJ, Eilers R. Authors reply: Importance of standardisation of HAI definitions in interpretation of international and/or multinational prevalence studies . Euro Surveill. 2012;17(37):pii=20270. Available online: http://www.eurosurveillance.org/ViewArticle.aspx?Articleld=20270

\section{To the editor:}

We would like to thank $M$ Cotter et al. for their comments regarding the application of uniform definitions for infections in the nursing home setting. In our article we indeed used a 'suspicion of infection', i.e. having at least one symptom or sign on the healthcare-associated infections in long-term care facilities (HALT) score list. In this first European HALT study it was decided to register signs and symptoms of disease separately so that Mc Geer criteria might be applied afterwards. Previously, Rothan-Tondeur et al argued that it is time to revise the Mc Geer criteria [1].

It is important to have uniform definitions not only for prevalence studies such as the HALT study, but also for incidence studies of infectious diseases in nursing homes. In the Netherlands we have a sentinel surveillance network for infectious diseases in nursing homes (SNIV) in place since January 2009. Within this network, on a weekly basis and for each participating nursing home, an elderly care physician or nurse practitioner records the number of gastroenteritis, probable pneumonia and influenza-like illness and urinary tract infections based on clinical criteria. In 2009 we adopted clinical definitions used by general practitioners for surveillance of influenza-like illness and probable pneumonia, and guidelines for gastroenteritis used in research in nursing homes since 2007, for the SNIV network (Box).

After half a year of surveillance in 2009 we evaluated the above definitions used in the SNIV network and compared them to the McGeer criteria [2]. For this evaluation we interviewed eight elderly care physicians, who in the Netherlands are in charge of medical care to nursing home residents and are medical doctors specialised in providing medical care to the elderly [3]. The focus of the interviews was the way the elderly care physicians diagnose infectious disease in these residents and the experiences thus far participating in the SNIV nursing home network. We concluded that the clinical view of the respondents on the infectious diseases under surveillance in the SNIV network very much agreed with the clinical definitions as used for the surveillance.

We see it as a challenge for the HALT-2 study to gather the experiences of the different European countries with the application of surveillance definitions for infectious disease. With $M$ Cotter et al. we hope that the HALT-2 study in 2013 addresses the deficit of uniform European definitions of infectious diseases in nursing homes. In particular, we find it important to consider the need to base future clinical criteria for surveillance definitions on ways in which physicians diagnose infectious disease in nursing homes.

References

1. Rothan-Tondeur M, Piette F, Lejeune B, de Wazieres B, Gavazzi G. Infections in nursing homes: is it time to revise the McGeer criteria? J Am Geriatr Soc. 2010;58:199-201.

2. McGeer A, Campbell B, Emori TG, Hierholzer WJ, Jackson MM, Nicolle LE, et al. Definitions of infection for surveillance in long-term care facilities. Am J Inf Control. 1991; 19(1): 1-7.

3. Schols JM. Nursing home medicine in The Netherlands. Eur J Gen Pract. 2005;11(3-4:141-3. 
Definitions used by the 'Sentinel surveillance network for infectious diseases in nursing homes', the Netherlands, since 2009

\section{Gastroenteritis}

The resident must have one of the following four conditions:

a) diarrhoea three or more episodes in $24 \mathrm{~h}$, deviating from normal for this person

b) diarrhoea and two of the following symptoms: fever, vomiting, nausea, stomach ache, abdominal cramps, blood or mucus in stool

c) vomiting and two of the following symptoms: fever, nausea, stomach ache, abdominal cramps, blood or mucus in stool

d) vomiting three of more episodes in $24 \mathrm{~h}$ (without other symptoms and vomiting is not related to the use of medication).

\section{Influenza-like illness}

The resident must meet the following conditions:

a) an acute start of symptoms and

b) at least one of the following systemic symptoms: fever or febrile feeling, malaise, headache, myalgia and

c) at least one of the following three respiratory symptoms: cough, sore throat, shortness of breath.

\section{Probable pneumonia}

The resident must have a suspected infection of the low respiratory tract, probably pneumonia, and must have at least one of the following symptoms:

a) tachypnoea, malaise, confusion, shortness of breath, cough (productive or improductive),

fever $>38^{\circ} \mathrm{C}$ or fever in the last 48 hours, pain in the chest (respiratory) and

b) with new focal (unilateral) abnormalities upon auscultation of the lungs

as they occur as change compared to the former situation and other likely diagnoses are excluded. 